\title{
Evaluating Aortic Stenosis Using the Archimedean Copula Methodology
}

\author{
Pranesh Kumar ${ }^{1,2}$ and Mohamed M. Shoukri ${ }^{1,3}$ \\ ${ }^{1}$ King Faisal Specialist Hospital and Research Centre \\ ${ }^{2}$ University of Northern British Columbia and \\ ${ }^{3}$ University of West Ontario
}

\begin{abstract}
In modeling and analyzing multivariate data, the conventionally used measure of dependence structure is the Pearson's correlation coefficient. However use of the correlation as a dependence measure has several pitfalls. Copulas recently have emerged as an alternative measure of the dependence, overcoming most of the drawbacks of the correlation. We discuss Archimedean copulas and their relationships with tail dependence. An algorithm to construct empirical and Archimedean copulas is described. Monte Carlo simulations are carried out to replicate and analyze data sets by identifying the appropriate copula. We apply the Archimedean copula based methodology to assess the accuracy of Doppler echocardiography in determining aortic valve area from the Aortic Stenosis: Simultaneous Doppler Catheter Correlative study carried out at the King Faisal Specialist Hospital and Research Centre, Riyadh, KSA.
\end{abstract}

Key words: Copula functions, dependence measure, Monte Carlo simulations, Pearson's correlation coefficient, rank correlations.

\section{Introduction}

In medical studies, there are several treatments to compare and/or repeated measurements over time or prognostic factors to consider which lead to the multivariate and multi-dimensional data sets. A statistical challenge is to find models of populations that have wide applicability to real data. The normal or Gaussian distribution is one such statistical model. The statistical theory and methods associated with the normal distribution have many desirable mathematical properties like simplicity, analytical manageability and easy estimation of its only parameter, the correlation matrix. However empirical evidence suggests that the use of normal/multinormal distribution is inadequate in many situations (Marshal, Naldi and Zeevi, 2003). Whenever the assumption of normality fails, the 
extreme endpoints are more probable than anticipated by normal distribution not only in marginals but also in higher dimensions. For example, Gross and Lam (1981) in studying the survival distribution considered a bivariate exponential model with their marginals exponentially distributed and not a bivariate normal model to analyze data on a group of patients with transient conditions such as pain from chronic illness. Therefore, the assumption that a given data set is modeled adequately by a normal distribution is extremely important and should be tested prior to analyzing data.

An important issue in modeling is the choice of the appropriate dependence measure. The commonly practiced dependence measure is the Pearson's correlation coefficient $(r)$. However correlation as a measure of dependence has several drawbacks (Embrechts, McNeil and Straumann, 1999; Frees and Valdez, 1998; Schweizer and Wolff, 1981; Schweizer, 1991). Correlation is not a complete description of dependence structure even when there is a straight-line relationship between two random variables. Correlation is a canonical measure of the stochastic dependence used with normal (elliptical) distributions. It is strongly affected by extreme endpoints. Independence of two random variables implies they are uncorrelated but zero correlation in general does not imply independence unless the distributions are multivariate normal. Correlation is not invariant under non-linear strictly increasing transformations of random variables. Other classical non-parametric measures of dependence are Spearman's rank correlation $\rho$ and Kendall's rank correlation $\tau$. Both $\rho$ and $\tau$ measure the degree of monotonic dependence while $r$ measures the degree of linear dependence only. The advantage of rank correlations $\rho$ and $\tau$ over $r$ is that these are invariant under monotonic transformations.

An alternative to measure the dependence structure is copulas which overcome the limitations of correlation as a dependence measure (Sklar, 1959; Nelson, 1999; Genest and Rivest, 1993; Joe, 2005; Marshall and Olkin, 1988). Copulas are relatively a new concept and have been applied frequently in survival analysis, risk management and actuaries. Copulas are functions that join or couple multivariate distribution functions to their one-dimensional marginal distribution functions where the one-dimensional marginals are uniform on the interval $[0,1]$. There are several advantages of using copulas as dependence measures like copulas allow modeling both linear and non-linear dependence, any choice of a marginal can be used and extreme endpoints can also be modeled. In this paper, we describe the copula based simulation methodology which can be used in analyzing medical studies. We illustrate the application by analyzing the patients' data from the Aortic Stenosis: Simultaneous Doppler - Catheter Correlative study (Fawzy et al.,1995). The Monte Carlo simulations are carried out to replicate and analyze the data sets. 


\section{Methodology}

\subsection{Study example}

The study example is taken from a research study (Fawzy et al.,1995) to assess the accuracy of Doppler echocardiography (DE) in determining aortic valve area (AVA). A prospective study consisting of 30 patients aged 17-82 years with a clinical diagnosis of aortic stenosis and with no or mild aortic regurgitation and with no or mild mitral regurgitation was undertaken at the King Faisal Specialist Hospital and Research Centre. All patients underwent simultaneous Doppler echocardiograpic (DE) and cardiac catheterization (CC) with dual catheters in the ascending aorta and left ventricle using the transeptal technique. The catheter maximum gradient, mean gradient and AVA using Gorlin's formula were calculated. The DE data consisted of maximum gradient, mean gradient and AVA by continuity equation using velocity integral (method 1) and using maximum velocity (method 2). In this paper, however, we have considered data from CC AVA (denoted as Y hereafter) and DE AVA method 1 (denoted as X hereafter). The main question of interest is whether or not the non-invasive Doppler echocardiography technique is as accurate in identifying and assessing the severity of aortic stenosis as validated by simultaneous catheterization.

\subsection{Copula functions}

Let the bivariate distribution of $\mathrm{DE}$ aortic valve area, $X$ and $\mathrm{CC}$ aortic valve area, $Y$ be $H(x, y)$ with marginal distributions $F(x)$ and $G(y)$. Then for uniform random variables $U$ and $V$ defined on $[0,1]$ (by using the probability transforms $U=F(X)$ and $V=G(Y))$, there exists a bivariate copula function or simply copula $C(u, v)$ such that

$$
H(x, y)=\operatorname{Pr}[X \leq x, Y \leq y]=C(F(x), G(y))=C(u, v)
$$

It is discussed by Embrechts, McNeil and Straumann (1999) that the correlation $r$ is only a limited description of the dependence between random variables, except for the multivariate normal distribution where the correlation fully describes the dependence structure. If $F(x)$ and $G(y)$ are continuous then $C(u, v)$ is unique, else $C(u, v)$ is uniquely determined on range of $F(x) \times$ range of $G(y)$. An important feature of copulas is that any choice of marginal distributions can be used since copula links univariate marginals to their full multivariate distribution. Hence copulas are constructed based on the assumption that marginal distributions are known and in practice, by estimating the marginal distributions from the sample. 
The two standard non-parametric dependence measures Kendall's $\tau$ and Spearman's $\rho$ are expressed in the copula form as:

$$
\begin{aligned}
\tau & =4 \int_{0}^{1} \int_{0}^{1} C(u, v) d C(u, v)-1 \\
\rho & =12 \int_{0}^{1} \int_{0}^{1} C(u, v) d u d v-3
\end{aligned}
$$

Kendall's rank correlation $\tau$ is estimated from the data $\left(x_{i}, y_{i}\right), i=1,2, \ldots, n$ using:

$$
\tau=\frac{2}{n(n-1)} \sum_{i<j} \operatorname{sign}\left[\left(x_{i}-x_{j}\right)\left(y_{i}-y_{j}\right)\right]
$$

It may be noted that the simple correlation $\mathrm{r}$ is not expressible in copula form. A special class of copulas termed as Archimedean copulas (Schweizer, 1991) is given by $C(u, v)=\phi^{-1}[\phi(u)+\phi(v)]$, for all $u, v \in[0,1]$ and where $\phi(\cdot)$ is a generator function containing parameter, $\theta$, such that for all $t \in(0,1), \phi(1)=0, \phi^{\prime}(t)<0$, i.e., $\phi(t)$ is decreasing function of $t$ and $\phi^{\prime \prime}(t) \geq 0$ i.e., $\phi(t)$ is convex. Oneparameter families of the Archimedean copulas with their generator functions are tabulated in Nelson, (1999, page 94). Examples of bivariate Archimedean copulas are given in Table 1 . The parameter $\theta$ is estimated from (2.4) and the relationship between $\theta$ and $\tau$ given in the last column of Table 1 . The parameter $\theta$ in each case measures the degree of dependence and controls the association between the two variables. When $\theta \rightarrow 0$ there is no dependence and if $\theta \rightarrow \infty$ there is perfect dependence. Schweizer and Wolff (1981) show that the dependence parameter $\theta$ which characterizes each family of Archimedean copulas can be related to Kendall's $\tau$.

Table 1: Bivariate Archimedean copulas, generator functions and Kendall's $\tau$.

\begin{tabular}{lccc}
\hline Copula & Generator $\phi(t)$ & $C(u, v)$ & Kendall $\tau$ \\
\hline Product & $(\mathrm{a})$ & $(\mathrm{b})$ & 0 \\
(Independent) & & & \\
Clayton & $(\mathrm{c})$ & $(\mathrm{d})$ & $(\mathrm{e})$ \\
Gumbel & $(\mathrm{f})$ & $(\mathrm{g})$ & $(\mathrm{h})$ \\
Frank & $(\mathrm{i})$ & $(\mathrm{j})$ & $(\mathrm{k})$ \\
\hline
\end{tabular}


The formulas in this table are given as follows:

$$
\begin{aligned}
& (\mathrm{a})=-\ln t \\
& (\mathrm{~b})=u \cdot v \\
& (\mathrm{c})=t^{-\theta}-1, \theta>0 \\
& (\mathrm{~d})=\left(u^{-\theta}+v^{-\theta}\right)^{-1 / \theta} \\
& (\mathrm{e})=\frac{\theta}{(\theta+2)} \\
& (\mathrm{f})=(-\ln t)^{\theta}, \theta \geq 1 \\
& (\mathrm{~g})=\exp \left[-\left\{(-\ln u)^{\theta}+(-\ln v)^{\theta}\right\}^{1 / \theta}\right] \\
& (\mathrm{h})=\frac{(\theta-1)}{\theta} \\
& (\mathrm{i})=-\ln \left[\frac{e^{-t \theta}-1}{e^{\theta}-1}\right], \quad \theta \in R \\
& (\mathrm{j})=-\frac{1}{\theta} \ln \left[1+\frac{\left(e^{-u \theta}-1\right)\left(e^{-v \theta}-1\right)}{\left(e^{-\theta}-1\right)}\right] \\
& (\mathrm{k})=1-\frac{4}{\theta}\left[1-D_{1}(\theta)\right],
\end{aligned}
$$

where $D_{k}(x)$ is the Debye function for any positive integer $k$, given by $D_{k}(x)=$ $\frac{k}{x^{k}} \int_{0}^{x} \frac{t^{k}}{e^{t}-1} d t$

For the given application data, the sample versions of measures of dependence can be expressed in terms of empirical copulas and corresponding empirical copula frequency function (Nelson, 1999, page 176).

Definition 1. Given $\left(x_{i}, y_{i}\right), i=1,2, \ldots, n$ a sample of size $n$ from a bivariate distribution, the empirical copula is $C(i / n, j / n)=$ [Number of pairs $(x, y)$ in the sample such that $x \leq x_{(i)}$ and $\left.y \leq y_{(j)}\right] / \mathrm{n}$, where $x_{(i)}$ and $y_{(j)}, 1 \leq I, j \leq n$ denote order statistics from the sample. The empirical copula frequency function is given by $C(i / n, j / n)=1 / n$ if $\left(x_{(i)}, y_{(j)}\right.$ is an element of the sample; 0 otherwise.

\subsection{Tail dependence}

Tail dependence refers to the amount of dependence in the tails of a bivariate distribution or alternatively the dependence in the corner of the lower-left quadrant or upper-right quadrant of a bivariate distribution. The general dependence structure, especially the dependence structure of extreme events, strongly influences the analysis and thus should be considered seriously during analysis. For two random variables $X$ and $Y$ with marginal distributions $F(x)$ and $G(y)$, the upper tail-dependence is defined as: 


$$
\lambda_{\text {upper }}=\lim _{u \rightarrow 1} \operatorname{Pr}\left[Y \geq G^{-1}(u) \mid X \geq F^{-1}(u)\right]
$$

and the lower tail dependence by symmetry as:

$$
\lambda_{\text {lower }}=\lim _{u \rightarrow 0} \operatorname{Pr}\left[Y \leq G^{-1}(u) \mid X \leq F^{-1}(u)\right]
$$

provided limits exist, for $u \in(0,1)$ and $F^{-1}(u)$ and $G^{-1}(u)$ are the inverse distribution functions of $X$ and $Y$ respectively. The distribution is upper (lower) tail dependent if $\lambda_{\text {upper }}\left(\lambda_{\text {lower }}\right)>0$ and upper (lower) tail independent if $\lambda_{\text {upper }}\left(\lambda_{\text {lower }}\right)=$ 0 .

\subsection{Copula and tail dependence}

The following representation shows that tail dependence is a copula property. An equivalent definition (for continuous random variables) of tail dependence in terms of a bivariate copula function $C(u, v)$ :

$$
\lambda_{\text {upper }}=\lim _{u \rightarrow 1} \frac{1-2 u+C(u, u)}{1-u}
$$

and

$$
\lambda_{\text {lower }}=\lim _{u \rightarrow 0} \frac{C(u, u)}{u}
$$

\subsection{Algorithm to generate Archimedean copulas}

The following algorithm generates random variables $(U, V)$ whose joint distribution is an Archimedean copula $C(u, v)$ with generator function $\phi(t)$.

1. Generate two independent uniform random variables $p$ and $q$ on $[0,1]$.

2. Set $t=K_{C}^{-1}(q)$ where $K_{C}$ is a copula function $C(u, v)$.

3. Set $u=\phi^{-1}[p \phi(t)]$ and $v=\phi^{-1}[(1-p) \phi(t)]$.

4. Find $x=F^{-1}(u)$ and $y=F^{-1}(v)$

5. Repeat $n$ times steps 1 through 5 to generate $n$ pairs of data $\left(x_{i}, y_{i}\right), i=$ $1,2, \ldots, n$.

For implementing the algorithm, the codes are given in Melchiori (2003). The expressions for copulas considered in this paper are worked out and are given in Table 2. 
Table 2: Algorithm implementation for Archimedean copulas.

\begin{tabular}{lccc}
\hline & Clayton & Gumbel & Frank \\
\hline$\theta$ & $(\mathrm{a})$ & $(\mathrm{b})$ & No closed form \\
$\phi(t)$ & $(\mathrm{c})$ & $(\mathrm{d})$ & $(\mathrm{e})$ \\
$\phi^{\prime}(t)$ & $(\mathrm{f})$ & $(\mathrm{g})$ & $(\mathrm{h})$ \\
$\phi^{-1}(t)$ & $(\mathrm{i})$ & $(\mathrm{j})$ & $(\mathrm{k})$ \\
$K_{C}$ & $(\mathrm{l})$ & $(\mathrm{m})$ & $(\mathrm{n})$ \\
$\left(\begin{array}{l}u \\
v\end{array}\right.$ & $(\mathrm{o})$ & $(\mathrm{p})$ & $(\mathrm{q})$ \\
\hline
\end{tabular}

The formulas in this table are given as follows:
(a) $=\frac{2 \tau}{1-\tau}$
(b) $=\frac{1}{1-\tau}$
(c) $=t^{-\theta}-1$
$(\mathrm{d})=(-\ln )^{\theta}$
(e) $=T=:-\ln \left[\frac{e^{-t \theta}-1}{e^{\theta}-1}\right]$
(f) $=-\theta \cdot t^{-\theta-1}$
$(\mathrm{g})=-\frac{\theta(\ln t)^{\theta-1}}{t}$
(h) $=\frac{\theta}{1-e^{t \theta}}$
(i) $=(1+t)^{-1 / \theta}$
(j) $=\exp \left[-t^{1 / \theta}\right]$
$(\mathrm{k})=-\frac{\ln \left(1-e^{-t}+e^{-t-\theta}\right)}{\theta}$
(l) $=t-\frac{t^{1+\theta}-t}{\theta}$
$(\mathrm{m})=t-\frac{t \ln t}{\theta}$
(n) $=t-\frac{e^{t \theta}-1}{\theta} \ln \left[\frac{e^{-t \theta}-1}{e^{-\theta}-1}\right]$
$(\mathrm{o})=\left(\begin{array}{c}\left(1+p\left(t^{-\theta}-1\right)\right)^{-1 / \theta} \\ \left(1+(1-p)\left(t^{-\theta}-1\right)\right)^{-1 / \theta}\end{array}\right)$ 


$$
\begin{aligned}
& (\mathrm{p})=\left(\begin{array}{c}
\exp \left(-\left(p(-\ln t)^{\theta}\right)^{1 / \theta}\right) \\
\exp \left(-\left\{(1-p)(-\ln t)^{\theta}\right\}^{1 / \theta}\right)
\end{array}\right) \\
& (\mathrm{q})=\left(\begin{array}{c}
-\frac{\ln \left(1-e^{-p T}+e^{-p T-\theta}\right)}{\theta} \\
-\frac{\ln \left(1-e^{-(1-p) T}+e^{-(1-p) T-\theta}\right)}{\theta}
\end{array}\right)
\end{aligned}
$$

\subsection{Which copula is the best?}

The first step in modeling and simulation is to identify the appropriate copula form. To identify the best copula for the given application data $\left(x_{i}, y_{i}\right), i=$ $1,2, \ldots, n$ we carry out the following steps (Genest and Rivest, 1993; Frees and Valdez, 1998):

1. Determine the pseudo observations $T_{i}=\left\{\right.$ Number of $\left(x_{j}<x_{i}\right)$ such that $x_{j}<x_{i}$ and $\left.y_{j}<y_{i}\right) /(n-1)$, and the empirical copula $K_{E}(t)=$ proportion of $T_{i} \leq t, 0 \leq t \leq 1$.

2. Choose that copula which minimizes the non-parametric distance measure $D M=\int\left[K_{C}(t)-K_{E}(t)\right]^{2} d K_{E}(t)$.

\section{Results}

\subsection{Application}

The scatter plot of data on the aortic valve area in $\mathrm{cm}^{2}$ determined by Doppler Echocardiology (AVADE) and Cardiac Catheterization (AVACC) from 30 patients aged 17-82 years is shown in Figure 1. The descriptive analysis indicates that (i) AVADE and AVACC both have departures from symmetry (non significant skewness coefficients being -0.191 and 0.141, respectively) and (ii) AVADE and AVACC both have tendency of the observations to cluster less and have shorter tails than those in normal distribution (nonsignificant kurtosis coefficients being -0.631 and -1.003 , respectively). To estimate the marginal distributions 


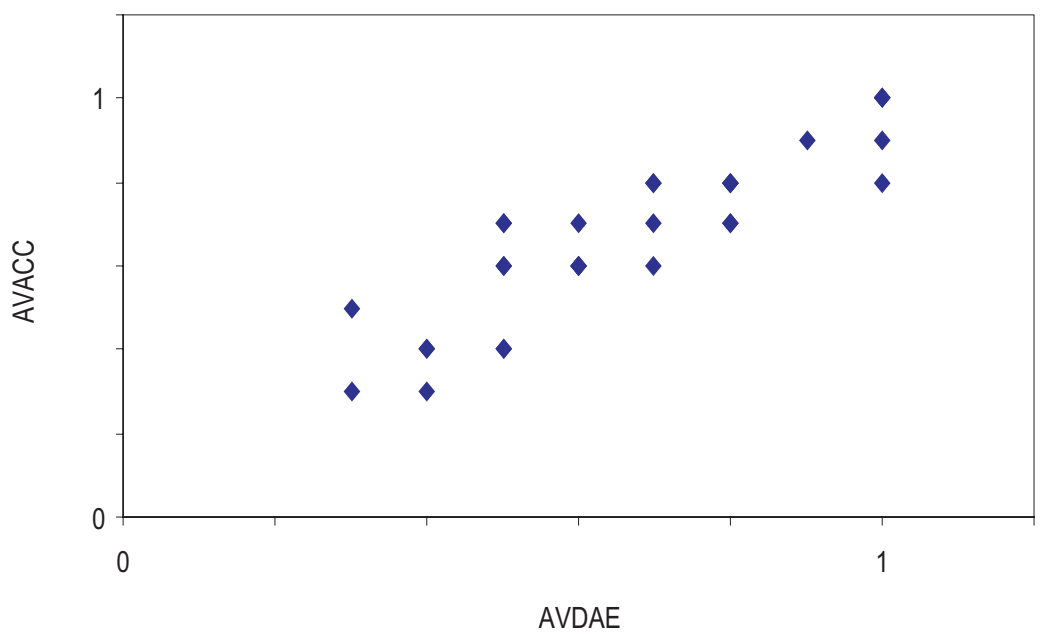

Figure 1: Aortic valve areas $\left(\mathrm{cm}^{2}\right)$ measured by Doppler Echocardiograph (AVADE) and Cardiac Catheter (AVACC).
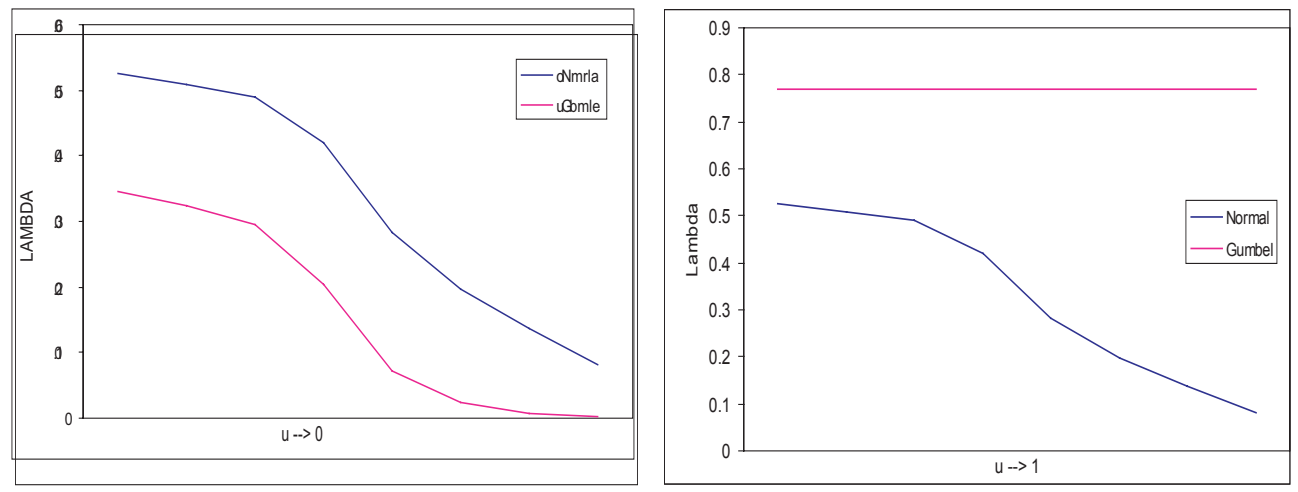

Figure 2: (a) Left panel: Lower tail dependence; (b) right panel: Upper tail dependence.

of AVADE and AVACC, we have noticed from their probability plots that both variables can be approximated by the normal distribution as well as gamma distribution since the points clustered around a straight line. The estimated normal and gamma distributions are: AVACC Normal (0.663, 0.217); AVADE $\sim \operatorname{Normal}(0.67,0.2)$ or AVACC $\sim$ Gamma $(9.316,14.045) ;$ AVADE $\sim$ Gamma (11.194, 16.707). As a conventional measure of dependence between AVADE and AVACC, we have calculated the correlation coefficient $r=0.701(p<0.0001)$ and non-parametric Kendall's rank correlation $\tau=0.893(p<0.0001)$. Based 


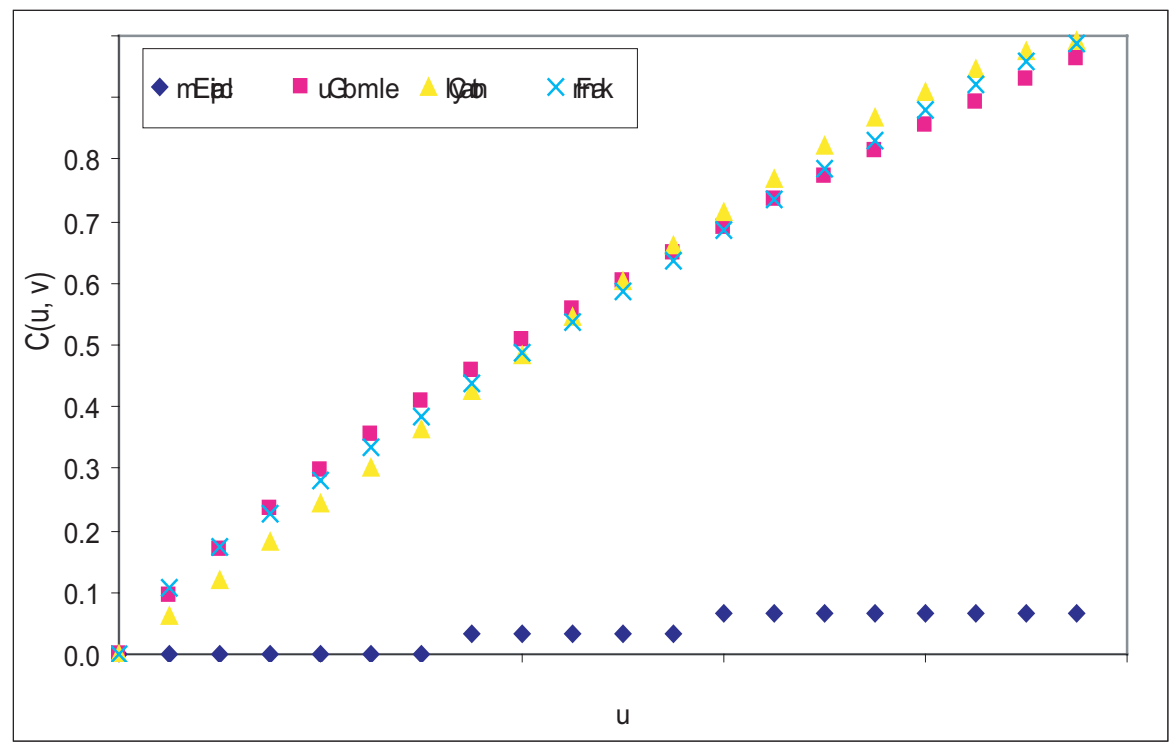

Figure 3: Which copula is better?

on the assumption of normality, the estimates (M.) and 95\% confidence intervals (CI) of mean and difference (D) of AVADE and AVACE in $\mathrm{cm}^{2}$ are:

$$
\begin{aligned}
M_{D E}= & 0.6633(95 \% C I=0.5855,0.7411) \\
M_{C C}= & 0.6700(95 \% C I=0.5983,0.7417) \\
D:= & M_{C C-D E}=00067 \\
& \quad \quad \quad(\text { one-tailed } p=0.35 \text { and } 95 \% C I=-0.0284,0.0417)
\end{aligned}
$$

For studying tail dependence, Figures $2 \mathrm{a}$ and $2 \mathrm{~b}$ are obtained from (2.7), (2.8), $C(u, v)$ and the relationship between $\theta$ and $\tau$ given in Table 1 . The Kendall's $\tau$ is estimated from the given data. It is noted from Figure 2a that when $u \rightarrow 0$ the lower tail dependence tends to zero for both - normal and Gumbel copulas. When $u \rightarrow 1$ Figure $2 \mathrm{~b}$ shows that the upper tail dependence for the normal copula tends to zero, however, it remains almost constant for the Gumbel copula. So, the tail dependence analysis indicates that the Gumbel copula have upper tail dependence but does not have the lower tail dependence whereas the normal copula have neither. Therefore, the conventional statistical analysis based on the normality assumption and correlation as the measure of dependence is not appropriate. A copula based analysis which models the tail dependence as well, is a right choice to do the analysis. 


\subsection{Simulation results}

Three copulas of the Archimedean family, namely Gumbel (1960), Clayton (1978) and Frank (1979) copulas, and empirical copula are estimated from the application data. These are given in Figure 3. The values of non parametric distance measure

$D M=\int\left[K_{C}(t)-K_{E}(t)\right]^{2} d K_{E}(t)$ for the Gumbel, Clayton and Frank copulas are respectively 6.6020, 6.9670 and 6.6960 from Figure 3, implying that the Gumbel copula is the best fit.

To compare the difference in mean aortic valve area measured by Doppler and Catheter, data are simulated using the Gumbel copula with the estimated marginal distributions, both normal and gamma. For $n=30$ patients, 50, 100 and 200 Monte Carlo simulations are performed to replicate data sets using the Gumbel copula. The VBA codes provided in Melchiori (2003) are used for executing the algorithm.

For the parameter mean difference of the aortic valve area measured by Doppler and Catheter, point estimates, 95\% confidence intervals and width of the confidence

Table 3: Estimates of difference in means of aortic valve area $\left(\mathrm{cm}^{2}\right)$ measured by Doppler and Catheter from the application and simulated data using the Gumbel copula with marginal distributions normal and gamma $(n=30$ patients).

\begin{tabular}{lccccc}
\hline & Simulation & Estimate & \multicolumn{2}{c}{$95 \%$ Confidence Interval (CI) } & Width \\
\cline { 5 - 6 } & & $D=M_{C C-D E}$ & Lower Limit & Upper Limit & of CI \\
\hline Application & & 0.0067 & -0.0284 & 0.0417 & 0.0701 \\
\hline Gumbel copula & 50 & 0.0050 & -0.0276 & 0.0376 & 0.0652 \\
(Gamma margins) & 100 & 0.0069 & -0.0299 & 0.0399 & 0.0658 \\
& 200 & 0.0078 & -0.0253 & 0.0410 & 0.0663 \\
\hline Gumbel copula & 50 & 0.0097 & -0.1667 & 0.1710 & 0.3377 \\
(Normal margins) & 100 & 0.0048 & -0.1652 & 0.1749 & 0.3401 \\
& 200 & 0.0090 & -0.1605 & 0.1798 & 0.3403 \\
\hline
\end{tabular}

intervals are presented in Table 3 . The results in Table 3 indicate that the noninvasive Doppler Echocardiography technique is as accurate in identifying and assessing the severity of aortic stenosis as the Catheterization.

It is interesting to note that the Gumbel copula with gamma marginals performed better than the Gumble copula with normal marginals. The width of the confidence intervals from gamma based Gumbel copula simulation is smaller than the width of the confidence interval from the given data using standard method 
and also those from the normal marginals based Gumbel copula simulation. Thus, the gamma distributions estimate marginal distributions better than the normal distributions in the given data. Another noteworthy indicative conclusion which is evident from Table 3 is that the confidence intervals and consequently their width remain more or less the same for 50, 100 and 200 simulations. Thus, it is not necessary to carry out a large number of simulations when using the copula based methodology.

\subsection{Copula versus correlation based methodology}

Tail dependence analysis and estimated marginals distributions of the AVADE and AVACC variables indicated the upper tail dependence and marginals to be non-normal distributions and hence the application of copula based methodology. For comparing two methods, the predicted aortic valve areas from both the copula and correlation based prediction models and actual data are plotted in Figure 4. As

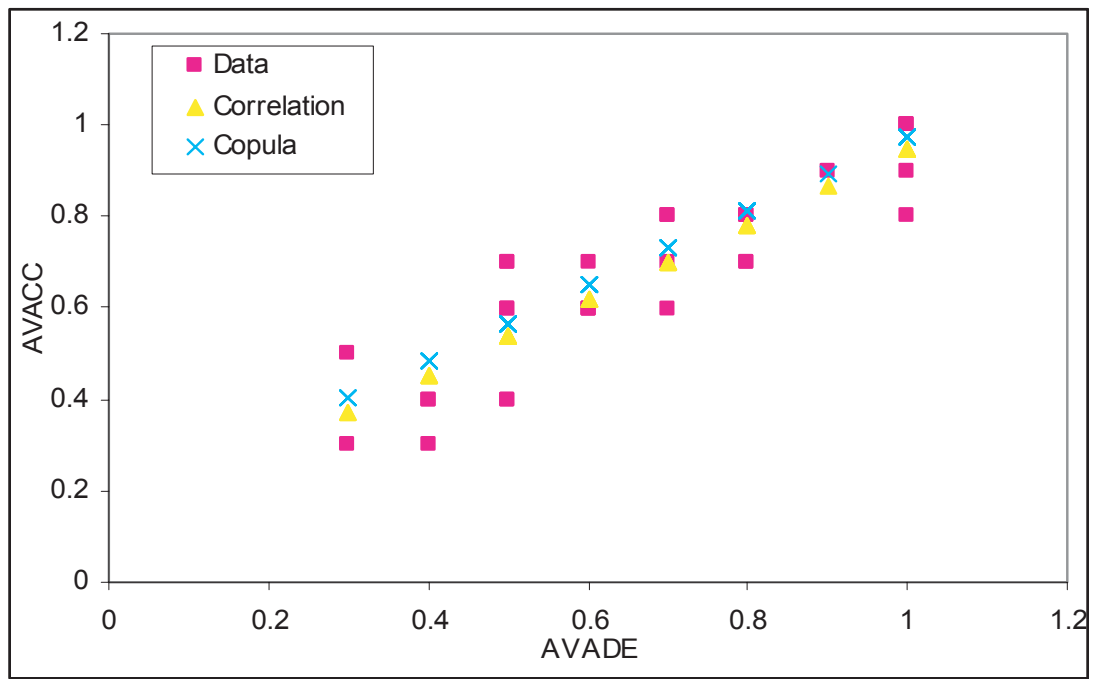

Figure 4: Predicted aortic valve areas using Gumbel copula and correlation based prediction models.

expected, the predicted aortic valve areas from both models are close, however, differences are noticeable for the measurements on the upper scale of the data. This is because of copulas being a more appropriate dependence measure than the correlation for the skewed endpoint data. Copula is capable of modeling the linear and non-linear endpoint behavior of the measurements in either or both directions. 


\section{Discussions}

In this paper, we envisage that the correlation coefficient is not a complete description of dependence structure even when there is a straight-line relationship between two random variables. An alternative to model the dependence structure is using copulas which overcome the limitations of the correlation. Copulas are functions that join or couple multivariate distribution functions to their onedimensional marginal distribution functions where the one-dimensional margins are uniform on the interval $[0,1]$. Copulas allow modeling both linear and nonlinear dependence. Using copulas, any choice of marginal distributions can be used and extreme endpoints can be modeled.

Based on a study undertaken at the King Faisal Hospital and Research Center to assess the accuracy of Doppler echocardiography as compared to the catheterization in clinical diagnosis of aortic stenosis in heart patients, we have described the copula based alternative methodology to model the dependence structure between risk-factors, to simulate the data and its application in analyzing the multifactor data. Since a number of families of copulas exist, this approach provides flexibility in modeling various categories of applications and simulating the data. In clinical trials or medical experiments, sample size is often an important consideration and is relatively small. Copula based methodology overcomes this limitation as well, because the algorithm can be used to replicate data for any number of patients. The suggested copula based methodology presented in this paper is simple and easy to implement.

\section{Acknowledgements}

Authors thank the referee for the valuable comments. Authors also thank the Research Centre, KFSH\&RC for sponsoring the research project RAC\# 2060 022 .

\section{References}

Clayton, D. G. (1978). A model for association in bivariate life tables and its applications in epidemiological studies of familial tendency in chronic disease incidence. Biometrika 65, 141-151.

Embrechts, P., Mcneil, A. J. and Straumann, D. (1999). Correlation and dependence in risk management: properties and pitfalls. In Risk Management: Value at Risk and Beyond (Edited by M. Dempster and H. K. Moffatt). Cambridge University Press.

Fawzy, M. E., Awad, M., de Vol, E., El-Deeb, F., Andaya, W. and Duran, C. (1995). Aortic stenosis: non-invasive assessment. Validation study by simultaneous Doppler 
catheterization. Technical Report, King Faisal Specialist Hospital and Research Center.

Frank, M. J. (1979). On the simultaneous associativity of $F(x, y)$ and $x+y-F(x, y)$. Aequationes Math. 19, 194-226.

Frees, E. W. and Valdez, E. (1998). Understanding relationships using copulas. North American Actuarial Journal 2, 1-25.

Genest, C. and Rivest, L. (1993). Statistical inference procedures for bivariate Archimedean copulas. Journal of the American Statistical Association 88, 1034-1043.

Gross AJ, Lam CF (1981). Paired observations from a survival distribution. Biometrics, $37: 505-511$.

Gumbel, E. J. (1960). Bivariate exponential distributions. Journal of the American Statistical Association 55, 698-707.

Gumbel, E. J. (1960). Distributions des valeurs extremes en plusiers dimensions. Publication of the Institure of Statistics, University of Paris 9, 171-173.

Joe, H. (2005). Parametric families of multivariate distributions with given marginals. Journal of Multivariate Analysis 46, 262-282.

Marshal, R., Naldi, M. and Zeevi, A. (2003). Comparing the dependence structure of equity and asset returns. RISK 16, 82-87.

Marshall, A. W. and Olkin, I. (1988). Families of multivariate distributions. Journal of the American Statistical Association 83, 834-841.

Melchiori, M. R. (2003). Which Archimedean copula is the right one? Yield Curve 37, 1-20.

Nelson, R. B. (1999). An Introduction to Copulas. Springer-Verlag.

Schweizer, B. (1991). Thirty years of copulas. In Advances in Probability Distributions with Given Marginals (Edited by G. Dall'Aglio, S. Kotz, G. Salinetti), 13-50. Kluwer Academic Publishers.

Schweizer, B. and Wolff, E. F. (1981). On nonparametric measures of dependence for random variables. Annals of Statistics 9, 879-885.

Sklar, A. (1959). Functions de repartition a $n$ dimensions et leurs merges. Publication of the Institure of Statistics, University of Paris 8, 229-231.

Received March 4, 2007; accepted March 24, 2007. 
Pranesh Kumar

Department of Biostatistics

Epidemiology and Scientific Computing

King Faisal Specialist Hospital and Research Centre

MBC \#03, P. O. Box 3354

Riyadh 11211, Saudi Arabia

kumarp@unbc.ca

Also:

University of Northern British Columbia

Prince George, BC

V2N 4Z9, Canada

Mohamed M. Shoukri

Department of Biostatistics

Epidemiology and Scientific Computing

King Faisal Specialist Hospital and Research Centre

MBC \#03, P. O. Box 3354

Riyadh 11211, Saudi Arabia

shoukri@kfshrc.edu.sa

Also:

Department of Epidemiology and Biostatistics

Schulich School of Medicine

University of Western Ontario,

London, Ontario, Canada 